\title{
EXPERIMENTAL INVESTIGATION OF STRESS AND STRAIN FIELDS IN A DUCTILE MATRIX SURROUNDING AN ELASTIC INCLUSION
}

\author{
E. E. NUGENT $\dagger$, R. B. CALHOUN and A. MORTENSEN \\ Department of Materials Science and Engineering, Massachusetts Institute of Technology, Cambridge, \\ MA 02139, USA
}

(Received 17 May 1999; accepted 23 November 1999)

\begin{abstract}
A method for measuring stress and strain distributions within a ductile material deforming by dislocational slip is developed. The method exploits the transparency and room-temperature ductility of silver chloride, and combines the techniques of photoelasticity and marker tracking. This method is used to investigate the deformation of an elasto-plastic ductile matrix surrounding an isolated stiff fiber, the grain size of the material being slightly smaller than the fiber length. The data are compared to predictions of finite element calculations which take the matrix to be an isotropic elasto-plastic von Mises continuum. It is found that this model does not fully capture all of the features of the experimental data. Data suggest that the cause for observed discrepancies is the strong influence exerted by grain boundaries and grain orientation on the distribution of stress and strain within the matrix. A comparison is also made between the data and predictions of the Eshelby equivalent inclusion calculation, to show that a far higher level of discrepancy results than with the finite element calculations; this is caused by the fact that the Eshelby equivalent inclusion calculation is essentially elastic and thus allows significant stress concentrations. (C) 2000 Acta Metallurgica Inc. Published by Elsevier Science Ltd. All rights reserved.
\end{abstract}

Keywords: Optical microscopy; Composites; Compounds (ionic); Photoelastic effects; Mechanical properties (plastic)

\section{INTRODUCTION}

The hard, elastic inclusion in a matrix deforming by dislocational slip is one of the most ubiquitous and important problems in micromechanics of deformation of materials. This problem is of practical interest in metal matrix composites such as aluminum combined with ceramic particles or fibers, in two-phase alloys such as pearlite or dispersion hardened aluminum, and in many nominally singlephase metals containing unwanted second phases such as the oxide inclusions in low-carbon steel.

Principal methods used to analyze the behavior of two-phase elasto-plastic materials are extensions of Eshelby's analysis of ellipsoidal inclusions in an elastic matrix [1-3], and finite element techniques, summarized in Refs [4]-[9] for metal matrix composites. Experimental research on this problem is

$\uparrow$ Present address: Choate, Hall \& Stewart, Boston, MA, USA.

$\$$ Present address: Department of Materials, Swiss Federal Institute of Technology, CH-1015 Lausanne, Switzerland.

$\S$ To whom all correspondence should be addressed. mostly focused on certain types of materials. Prominent is the considerable work on dispersion hardened metals, summarized, for example, in Refs [10] and [11]. Dispersion hardened metals are well understood because transmission electron microscopy (TEM) provides a powerful tool for investigation of the substructure that evolves as metal deforms around elastic inclusions below approx. $1 \mu \mathrm{m}$ in diameter. It is now well known from experiment that, to explain the mechanical behavior of dispersion or precipitation hardened metals, analysis must go beyond continuum elasto-plasticity to include the influence of the dislocational nature of plastic flow.

Comparatively fewer experimental data exist for the case where second-phase particles are above about $1 \mu \mathrm{m}$ in diameter. The difficulty with metallurgical examination of plasticity near such inclusions arises in part because these second-phase particles are too large to be fully contained in a thinned electron-transparent sample of the material. Transmission electron microscopy then provides a view of only a small portion of the matrix near the inclusion, which is in addition subject to free-surface effects. Furthermore, with larger inclusions, the 
dislocation structures obtained by transmission electron microscopy must be interpreted with some caution because residual stresses are present in the composite [12], or because ion-milling produces thermal stresses of sufficient magnitude to cause extraneous dislocation motion and emission during thinning (whether conducted with or without a cold stage) $[12,13]$. For these reasons, only a few TEM studies of metal matrix composites can be deemed relatively free of artifacts, either because jet polishing was used to thin the matrix (e.g. [13-16]) or because dislocations were decorated by precipitation before thinning $[17,18]$.

In order to provide a more global view of matrix deformation and/or to alleviate problems that can arise during thinning, alternate techniques have been used to study the plastic deformation of metals near larger inclusions. These include etch-pit studies to characterize low density dislocation structures near second phases [19, 20], conventional or synchrotron X-ray diffraction as well as neutron diffraction studies, of as-processed (e.g. [21, 22]) or strained samples (e.g. [23-25]), electron channeling studies in the scanning electron microscope $[12,26$, 27], and surface strain measurement using either stereoimaging techniques [28-31], pre-coated microgrid displacement measurements [32-37], or tracking of microstructural features [38].

Despite their respective advantages, few of these alternate techniques provide microstructural information that can be correlated critically with current micromechanical analysis results. Etch pit studies are restricted to measurement of dislocation structures at low dislocation densities at a free surface. Electron channeling and transmission electron microscopy both sample material within roughly one micrometer from a free surface. Additionally, if data must be compared with micromechanical calculations, both techniques require a calibration of diffraction or dislocation patterns using strained unreinforced samples, which makes implicitly the assumption that there is a one-to-one correlation between substructure and local effective strain. Xray and neutron diffraction techniques suffer, respectively, from poor penetration depth and from poor three-dimensional spatial resolution, and furthermore often require a fine-grained matrix. Finally, although local deformation techniques have been extended to provide strain measurements at a truly microscopic scale, with metallic samples these techniques are all restricted to deformation at free surfaces.

We present in what follows a method which alleviates these limitations by providing both stress and strain data within the bulk of an elasto-plastic material. We then use this method to investigate the micromechanics of elasto-plastic matrix deformation around fibrous inclusions $250 \mu \mathrm{m}$ in diameter.

The method we present is based on the use of sil- ver chloride, a well-established transparent metal analog. Like a metal, silver chloride deforms by dislocation creation and motion and is capable of considerable plastic deformation, to such an extent that it has been called "transparent metal" by Nye [39].

Much is known about silver halides since these compounds are used industrially as photosensitive emulsions on photographic film; a wealth of information can be found in general reference books [40, $41]$, photography monographs $[42,43]$ or materials science series [44, 45]. Many typically metallic phenomena have been reported for the deformation of silver chloride: deformation by slip due to dislocation movement, strain-hardening, texture, slip bands, Lüder's bands, strain aging, necking, ductile fracture, ductile-brittle transitions, internal friction, fatigue, diffusion controlled creep, recovery and recrystallization. Two additional features of silver chloride make it attractive for the experimental investigation of elasto-plastic microdeformation: it is transparent, and stress-birefringent.

We begin with a presentation of the experimental procedure developed to generate data on stress and strain distributions around a fiber embedded in silver chloride under stress. We then compare data with results from two of the most commonly used methods in the micromechanical analysis of metal matrix composites, namely elasto-plastic finite element analysis and the Eshelby equivalent inclusion method.

\section{EXPERIMENTAL PROCEDURES}

\subsection{Specimen fabrication}

Since silver chloride is highly reactive, all sample fabrication and testing was performed in a cleanroom. Silver chloride also dissociates in the presence of light with a wavelength below $430 \mathrm{~nm}$ [46], so all work was performed under darkroom conditions. Particular care was taken to avoid exposure of samples to white light before any heat treatment, since small silver centers may be formed, which then grow during heat treatment into large silver precipitates which obscure viewing during testing [44, 47].

Silver chloride cuttings of $99.999 \%$ purity were obtained from Solon Technologies (Solon, OH). These were cleaned in a chlorine gas saturated solution, and then rinsed once in full-strength $(37 \%)$ $\mathrm{HCl}$ and three times in boiling high-purity distilled water. Once cleaned and dry, the cuttings were rolled in a hand rolling mill between two sheets of tungsten (one of the few metals which does not react with silver chloride [47]) to a thickness of $500 \mu \mathrm{m}$, and cut with a razor blade into 19-mm squares. The silver chloride squares were then cleaned again in the chlorine solution.

Strain mapping experiments were conducted on samples containing an internal array of very thin 
silver markers. These markers were created photolytically on one $\mathrm{AgCl}$ square at this stage of the process. To create the markers, the silver chloride was placed on a glass slide, and a small square of 1000 line-per-inch nickel mesh (purchased from Ladd Research, Williston, VT) glued with cyanoacrylate to a quartz slide was placed atop it and taped down in close contact with the silver chloride. A pinhole camera was used to focus the UV light from an unshielded mercury vapor lamp onto the sample from one to two hours. This process caused the formation of small and very thin square regions of silver precipitates at the surface of the silver chloride plate.

A variety of possible reinforcing fibers was tested for use in the silver chloride matrix composites. Nearly all materials that were tested either reacted with the silver chloride, or formed voids at the fiber ends when the sample was deformed in tension, indicating an insufficient interface strength. The only suitable candidate material found was pure titanium.

Reinforcing fibers were made from $99.7 \%$ titanium wire of diameter $250 \mu \mathrm{m}$, purchased from Aesar (Ward Hill, MA). The as-received titanium wire was first cleaned in boiling water, then straightened by stretching into the plastic deformation regime. It was then cut with a pair of scissors into lengths of 3-6 mm. To flatten the ends of the fibers, each fiber was inserted into a capillary tube having a $250-\mu \mathrm{m}$ inner diameter, and the assembly was polished flat at each end using 400 and 600 grit alumina paper.

Once the silver chloride squares and the titanium fibers were prepared, the sample was assembled and diffusion-bonded in a stainless steel die lined with

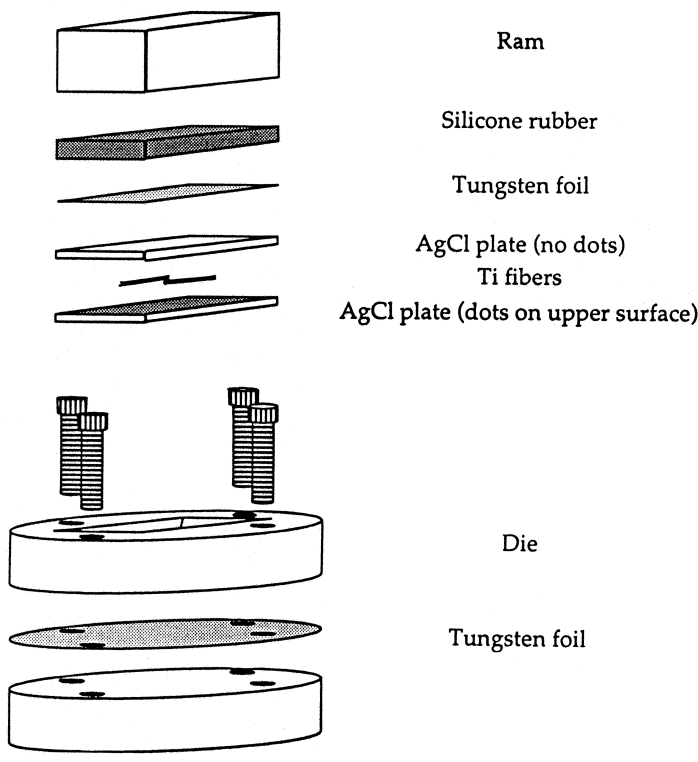

Fig. 1. Layup for diffusion bonding of specimens. tungsten foil, as described in Fig. 1. Diffusion bonding of the two sample halves was performed using a hot press fitted within a floor standing Instron Model TT-B tension/compression apparatus equipped with a Leybold TMP150 V DN100 turbomechanical vacuum pump and an induction heating coil, described in Ref. [48]. Details and parameters of the diffusion bonding process are given in Ref. [49].

This process yielded a 1-mm-thick square of silver chloride containing two parallel titanium fibers and, if desired, a grid of thin silver precipitates located exactly at the fiber midplane. Photoelastic examination of a sample immediately after diffusion bonding indicated the presence of residual stresses, particularly around the fiber: these are manifest as bright areas around the fiber under dark field circular polarization conditions. Because of the low melting temperature and high purity of the silver chloride used, recovery occurs at room temperature; after two days, samples become completely dark under dark field circular polarization. The sample was then cut with a razor blade into two tensile coupons, each 3-7 mm wide, $19 \mathrm{~mm}$ long, and con-

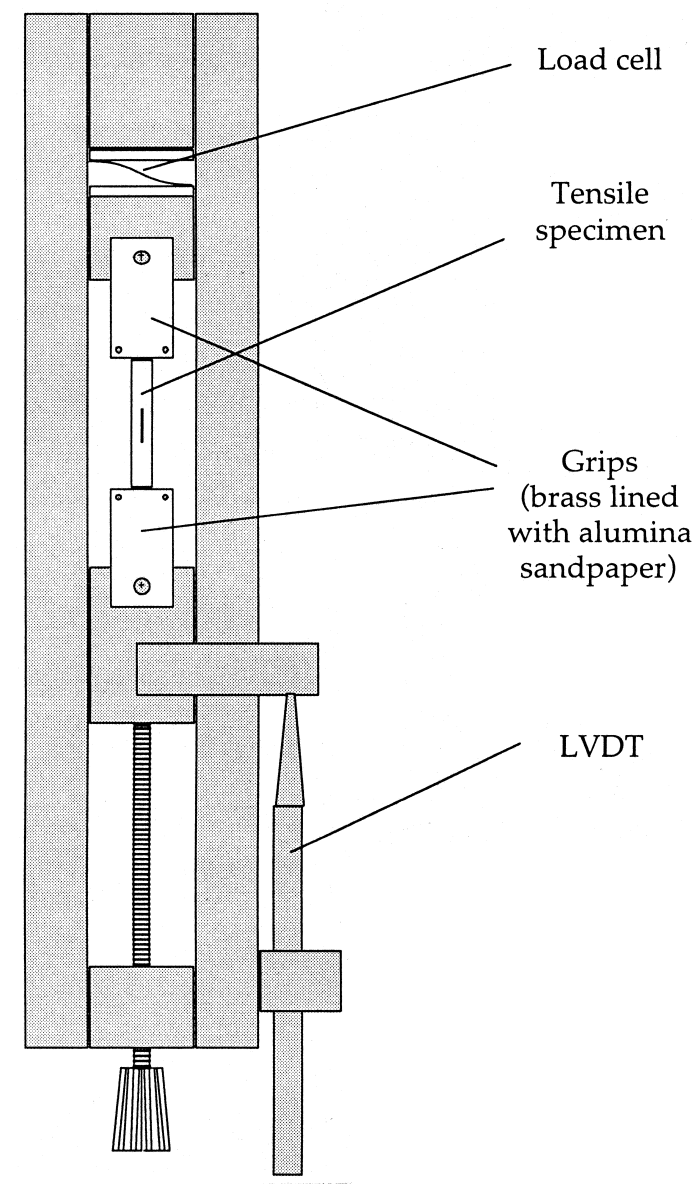

Fig. 2. Schematic of strain stage for tensile testing in the optical microscope (not to scale). 
taining one fiber. These were allowed to recover again at room temperature for two more days. The grain size of the specimens was generally on the order of 400-1000 $\mu \mathrm{m}$, although occasionally grains as large as $3000 \mu \mathrm{m}$ were found.

\subsection{Tensile tests}

To determine the flow properties of $\mathrm{AgCl}$ in the specimens, three unreinforced coupons cut from diffusion bonded samples were tested in tension.

A small screw-driven tensile stage was constructed so that samples could be deformed parallel to the sample axis under the microscope (Fig. 2). The load was measured using a $100 \mathrm{~N}$ load cell, and the displacement was measured with an LVDT. This latter measurement included the displacement of the load cell and hence overestimated the specimen elongation. The LVDT was therefore used chiefly to detect the onset of yield during testing.

All tests were conducted in a Zeiss G42-110-e Axioskop transmission optical microscope (Oberkochen, Germany). Data were recorded using a Dage-MTI CCD-72 camera (Michigan City, IN), connected to an Apple Macintosh IIci computer running IP Lab Spectrum 3.1 (Scanalytics, Vienna, VA). All photographs were taken using $546 \mathrm{~nm}$ green light, produced by a Zeiss $\sharp 467807$ filter.

When recording photoelastic data, photographs were taken at a magnification of $25 \times$ under four polarization conditions: bright field circular polarization, dark field circular polarization, bright field plane polarization, and dark field plane polarization. Theoretically, only one circular and one plane polarization image are needed to capture all accessible information about the integrated stress field; however, collection of the four modes allows for the compensation of spatial variation in sample clarity. In most cases, photos needed to be taken at three or four locations in order to capture all of the fiber and its immediate surroundings.

Strain marker data were recorded at a higher magnification of $100 \times$ using unpolarized light. Capturing the fiber and its immediate surroundings at this magnification required $20-25$ photos per specimen per strain increment. This process took about 10-15 min at each load level at which data were collected. At higher loads, the specimen relaxed slightly, so that the applied load dropped by $1-2 \%$ during each round of data collection.

For all tests, the sample was first imaged in the unloaded state. Then a small load was applied and the sample was imaged again. This process was repeated, using approximately constant load increments of $5 \mathrm{~N}$, until the displacement (measured using the LVDT) required to produce a given load step increased significantly, indicating that bulk yielding had initiated. At this time, images were captured at roughly constant-displacement incre- ments on the order of $0.05 \mathrm{~mm}$ until the sample began to neck visibly or to tear.

\subsection{Interpretation of photoelastic data}

As a cubic solid, unstressed silver chloride does not rotate polarized light. The magnitude of the optical rotation is proportional to the difference in orthogonal stress axes resolved onto the plane normal to the light propagation direction, integrated through the sample thickness. Under plane stress conditions, the stress state is readily computable from the resulting photoelastic image.

In principle, axisymmetric stress states are also computable by integration from a known far-field stress state. In practice, however, polycrystalline silver chloride composites deviated substantially from perfect axial symmetry and computation of the stress state from the photoelastic image was not possible. Therefore, theoretical photoelastic images were computed from the stress states predicted by the FEM and Eshelby models, for direct comparison with those produced experimentally. Differences between the theoretical predictions and the experimental results are readily discerned by this method, which also features high spatial resolution.

\subsection{Processing of marker images to extract displacements and strains}

All experiments were conducted such that the tensile axis coincided to the extent possible with the fiber axis. The printed grid was often not perfectly aligned with the fiber axis, and its initially square arrangement was deformed during diffusion bonding. For these reasons, and because the number of printed points was large (typically in excess of 10,000 points were tracked), dot tracking and image analysis techniques conventionally used with perfectly square grids aligned along the tensile axis [34, 50] could not be used in this study.

The problem of calculating the strain field in the midplane was broken into four parts: (i) identifying the marker locations in each image, (ii) assembling images surrounding a fiber at the same stress level, (iii) tracking marker movement in assembled images from one stress level to the next, and (iv) using the displacement field thus measured to calculate the strain field.

2.4.1. Marker location identification. Variations in the amount of silver precipitation within different grains, and sometimes out-of-focus inclusions which cast shadows on the fiber plane, caused variations in overall brightness across samples. Hence, if markers were traced by simply taking a fixed threshold brightness value across the sample, their size would vary significantly. Simple thresholding was therefore deemed inadequate, and an alternate procedure was developed in order to render the general brightness 


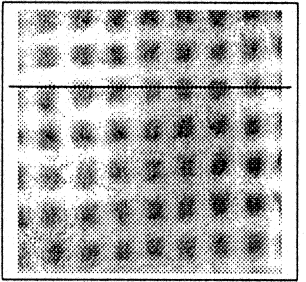

a

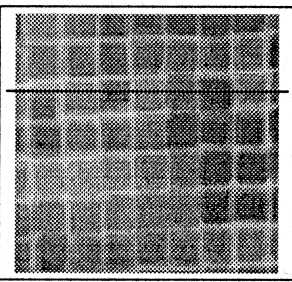

C

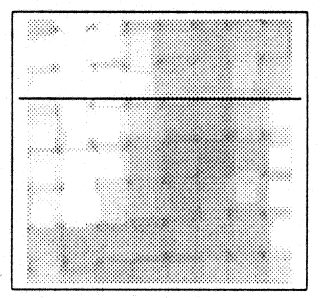

e

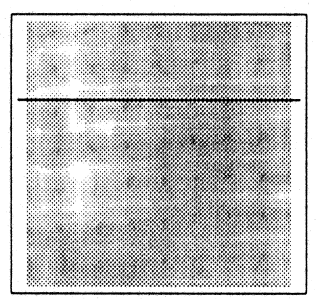

g

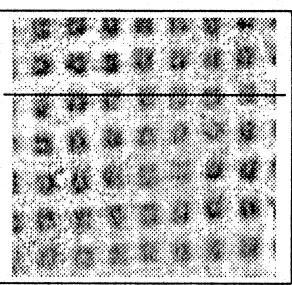

i

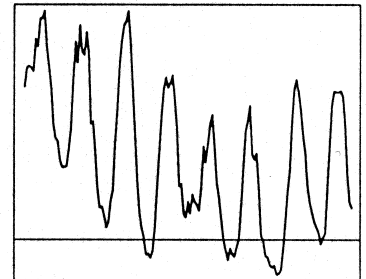

b

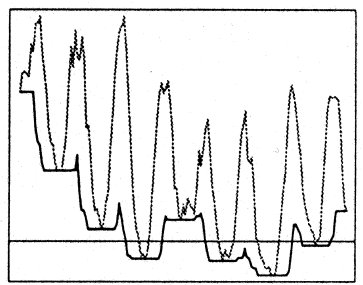

d

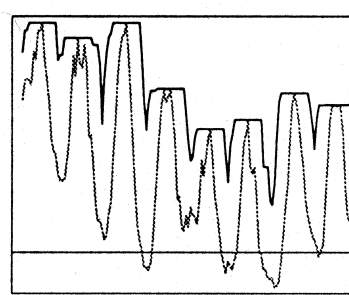

f

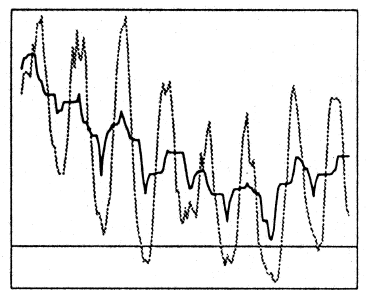

$\mathrm{h}$

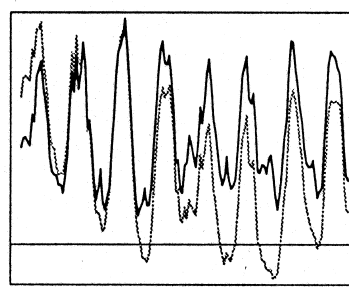

j
Fig. 3. Morphological image processing; (a) shows a small segment of a typical specimen image, (b) is a plot of the intensity in (a) along the indicated line; (c) and (d) represent eroded versions of (a) and (b) [the dotted line in (d) is the curve from (b)], while (e) and (f) represent dilated versions of the same data; $(\mathrm{g})$ and $(\mathrm{h})$ are the background data calculated by taking the mean of (c) and (e) and of (d) and (f), respectively; (i) and (j) represent the final corrected image obtained by subtraction of the background (g) and (h) from the original image in (a) and (b), and now featuring a uniform average intensity level. uniform over the sample before delineating markers by thresholding.

To this end, a hybrid morphological background subtraction procedure was used. This process was chosen because it allows features of a known size and shape to be easily distinguished [51]. This background subtraction process is illustrated in Fig. 3; details of the procedure are given in Ref. [49]. Results of the process were found to be reproducible, and, by visual inspection, to be in good accord with the original images; Fig. 4 provides an example. The accuracy of this method of determining marker position was estimated by comparing results for corresponding points on overlapping portions of independently processed neighboring images. The precision of centroid position detection was found to be about 0.5 pixels, corresponding to about $0.6 \mu \mathrm{m}$.

2.4.2. Combined image assembly. The Dage CCD camera used in this study was capable of capturing an image measuring $640 \times 480$ pixels. In order to obtain images of sufficient detail, it was necessary to capture 20-25 separate images of the fiber region and assemble them into one large combined image. The images were captured at each stress level progressing clockwise around the fiber, with an overlapping region spanning roughly $20 \%$ of the area of each individual image. One marker present in each overlapping region was identified and its approximate position recorded in both images in which it appeared. These data were used to estimate the relative positions of each neighboring pair of images in the combined picture.

The position of each individual image within the aggregate image was then calculated by changing the value of their coordinates so as to create the greatest degree of overlap between clearly identical points across the two images. To this end, a translation was deemed sufficient, as no rotation is introduced by the microscope stage between successive images. This translation was determined using an algorithm constructed to take account of all overlapping points across the two images. This was deemed necessary because the shape and centroid location of each dark feature in neighboring images was subject to variability after image processing, this variability resulting from variations in the relative position of the pixel grid, as well as small optical aberrations near image edges. The appropriate translation of marker grid positions in the new image was calculated using Mathematica 2.2.2 (Wolfram Research, Inc., Champaign, IL), according to an algorithm presented in detail in Ref. [49].

2.4.3. Marker movement tracking. The final step in calculating marker displacements was the matching of corresponding markers at successive stress levels. This calculation was again performed using Mathematica 2.2.2, and the relevant code is given 
and described in Ref. [49]. The algorithm essentially comprised the following steps.

- A few markers clearly present and identifiable at all stress levels were tracked manually. For each of the individual images captured in the zerostress state, one such flagged marker was selected, and this marker's position was recorded in each of the combined images.

- For computational efficiency in following steps of the procedure, the marker position data for each combined image were partitioned into smaller subsets of the about 11,000 total marker points present in each combined image. These subsets were defined by dividing the total sampled area into two-dimensional Voronoi cells defined around each of the markers manually selected, and enlarging these cells by inclusion of markers within $25(s-1)$ pixels of their border where $s$ is the number of load steps applied. This enlargement of the Voronoi cells after the onset of sample deformation was produced to ensure that all markers in a given subset appeared in all subsequent corresponding subsets despite strain gradients that may exist between neighboring cells.

- Once the sets corresponding to each deformation increment had been thus partitioned, these were analyzed so as to calculate local displacements. To this end, markers in each subset were first sorted in ascending order of absolute distance from the manually flagged marker. Then, for each marker, another marker was searched within a 5-pixel radius of the position in which it would be located if its displacement were the same as that of its nearest neighbor in the already matched set of markers for the load in question. If exactly one marker met this condition, it was deemed identified and added to the matched set of markers. If zero or multiple points met this condition, the marker was considered to be unidentifiable at that load level and a placeholder was inserted in the list of matched marker points. If such placeholders had to be inserted for two consecutive load levels, the point under consideration was discarded across all strain increments. Otherwise, the newly calculated set of matched points, containing occasional isolated placeholders in some cases, was added to the list of such sets and the procedure was performed again for the next marker.

- Once all markers had been processed across all strain increments, the set of displacements generated was visually examined for discontinuities. If it appeared that a group of markers had been misidentified, another marker in the vicinity of the misidentified group was manually identified, and the entire calculation was redone.

- As a final check to ascertain that no point had been misidentified, the entire list of matched markers was searched to determine whether any two initially distinct markers had been matched to the same marker in any subsequent list. If any such markers were found, both were discarded from the data set.

2.4.4. Strain map construction. Once the set of marker centroid displacements had been calculated, the overall displacement and strain fields could be determined for each global strain level. To calculate the distribution of displacement and strain, the data collected from the tracked irregularly spaced marker centroids were first transformed to data for points located along a strictly square grid with a 50-pixel spacing. This was achieved by extrapolation using displacements of marker points in the vicinity of the square grid points. To this end, for each point on the square grid, the displacement values for all marker centroids within a 35-pixel radius were extracted. If there were at least five such markers and their mean position was within 15 pixels of the grid point, a biquadratic fit was applied to each of the two displacement values of neighboring markers thus identified.

Values of the two displacement coordinates at the

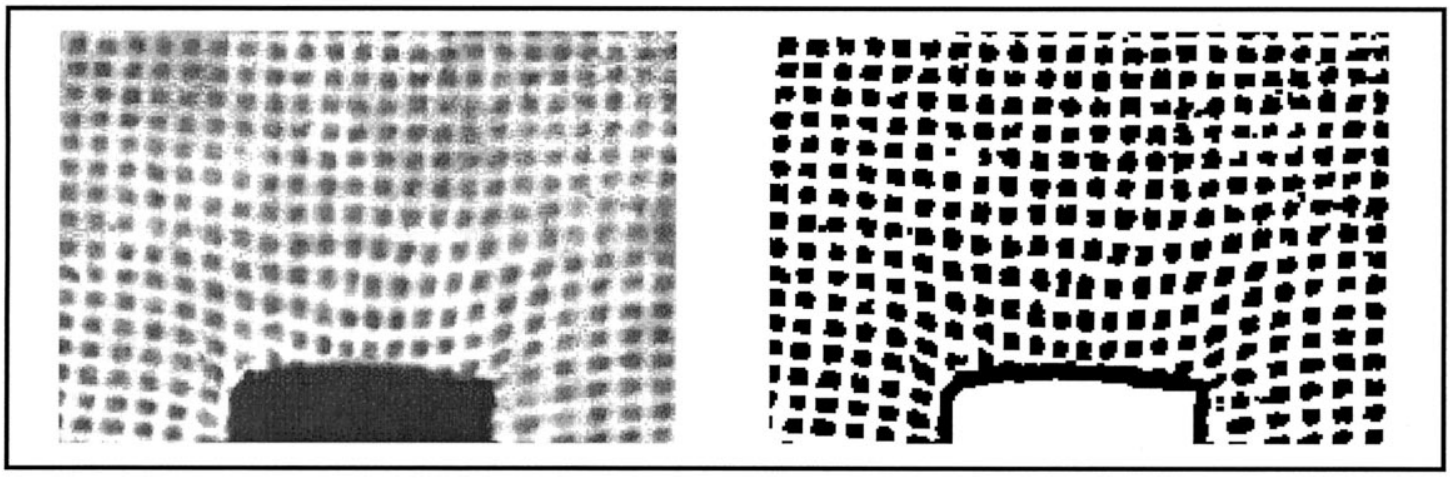

Fig. 4. Result of morphological image processing: image at left-hand side shows a direct picture of markers surrounding the tip of a fiber, while the right-hand side image shows the same markers after image processing as depicted in Fig. 3. The result is an image featuring sharpened and equisized dots. 
square grid point were derived from the value of the two biquadratic fits at that point. Values of the three strain components at that square grid point were calculated using the derivatives of the displacement fits at that point. Strains were calculated using the Green-Lagrange strain formulation:

$$
\left.\varepsilon_{i j}=\frac{1}{2}\left[\left(u_{i, j}+\delta_{i j}\right)\left(u_{j, i}+\delta_{i j}\right)-\delta_{i j}\right]\right] .
$$

\section{RESULTS}

\subsection{Bulk tensile testing of unreinforced $\mathrm{AgCl}$ specimens}

Since the specimens had length/width ratios substantially less than the minimum value of 4 required for sheet-type tensile specimens in the ASTM standard for tensile testing [52], it was assumed that the specimens deformed primarily in plane strain. Resulting curves of measured von Mises effective stress versus von Mises effective strain are given in Fig. 5. It is seen that silver chloride exhibits linear hardening after yield, with a relatively reproducible constant hardening coefficient of about $42 \mathrm{MPa}$. The ratio of the shear modulus to the hardening coefficient is therefore about 270 , which is similar to the value of 250 quoted for pure aluminum [53].

The values of the yield stress and work hardening rate measured here are significantly lower than most literature values, even for well-annealed silver chloride [44]; we attribute this mostly to a higher purity of the spectroscopic grade $\mathrm{AgCl}$ used in this study.

\subsection{Photoelastic and marker tracking specimens}

Table 1 summarizes the nature and experimental conditions for the four samples of this work.

3.2.1. Strain. Marker tracking data for the top and bottom halves of Sample 1 are given in Fig. 6 . The figures give color contours of strain parallel to the fiber axis. "Holes" in the strain maps represent areas where insufficient displacement data were available to calculate strains. The highly localized spikes in the strain data (appearing as red spots on these longitudinal strain plots) are due to extrapolation errors. Closer examination shows that these spikes generally occur in areas where most of the displacement data from which strain was calculated lie to one side of the point at which the strain was calculated. Also, strain values could not be calculated in a narrow band adjacent to the fiber, because this calculation would have required more displacement data than were available to perform an accurate numerical differentiation. The width of this band, $25 \mu \mathrm{m}$, is about the same as one marker spacing.

The observed deformation patterns feature higher

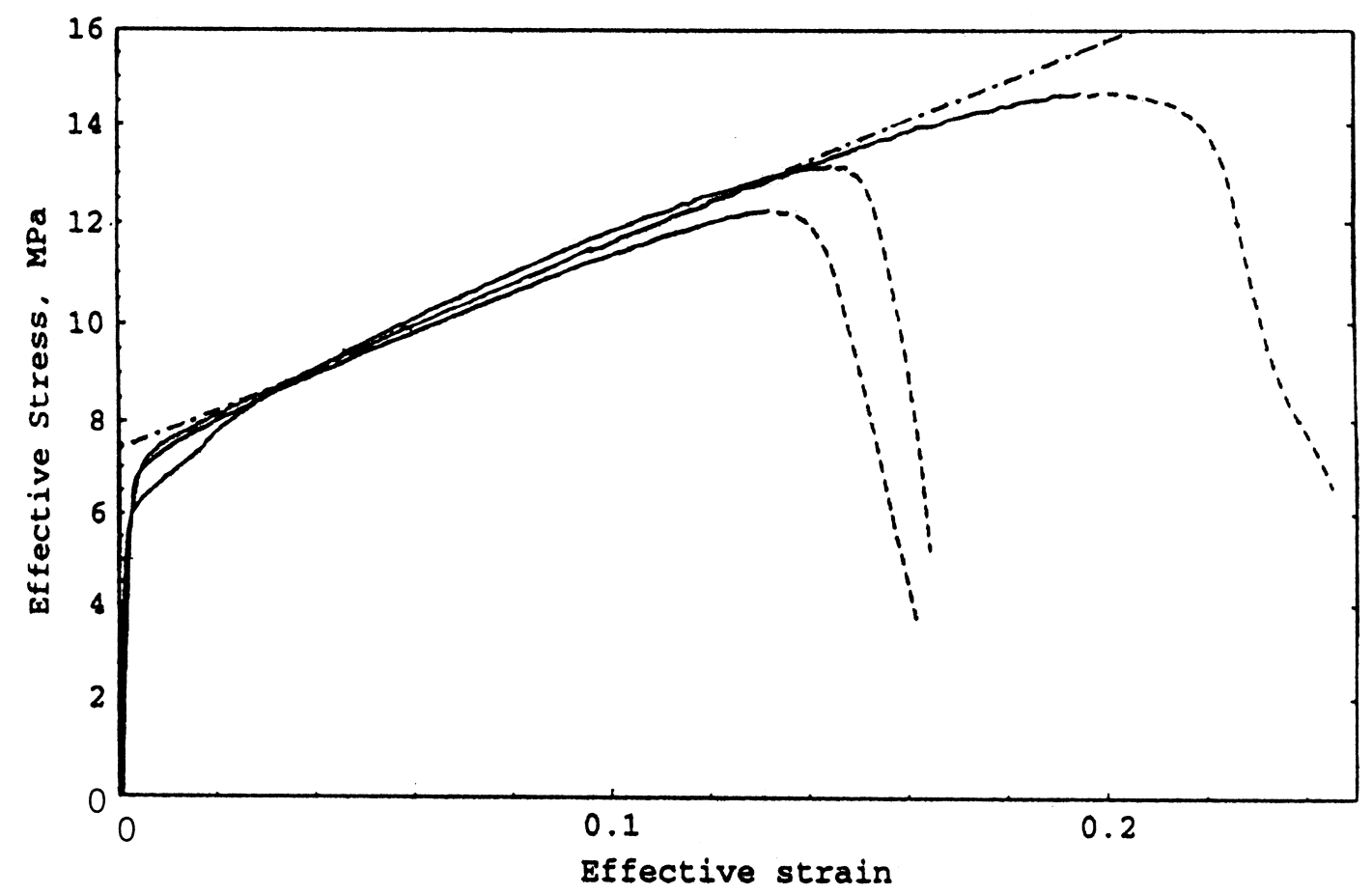

Fig. 5. Effective von Mises stress-strain curves for three tensile specimens. Dotted portions of curve indicate data gathered after maximum load. Dot-dashed line represents linear fit to data for strains between 0.03 and 0.13 . 
Table 1. List of samples, with type of data collected

\begin{tabular}{lccccc}
\hline Sample & $\begin{array}{c}\text { Marker data } \\
\text { collected }\end{array}$ & $\begin{array}{c}\text { Photoelastic } \\
\text { data collected }\end{array}$ & $\begin{array}{c}\text { Number of } \\
\text { load steps }\end{array}$ & $\begin{array}{c}\text { Maximum stress } \\
\text { (MPa) }\end{array}$ & $\begin{array}{c}\text { Maximum strain, as calculated by } \\
\text { finite element analysis (\%) }\end{array}$ \\
\hline 1 & Yes & No & 4 & 8.4 & 1.1 \\
2 & No & Yes & 5 & 9.0 & 1.8 \\
3 & Yes & No & 7 & 8.4 & 1.1 \\
4 & Yes & Yes & 6 & 9.5 & 2.4 \\
\hline
\end{tabular}

levels of strain near the fiber tips compared with the fiber sides; this is as expected, and it shows that the fiber does exert an influence on plastic flow of the surrounding matrix. Similar longitudinal, transverse, and shear strain plots were generated for all samples containing markers.

Overall features of these data are similar across all samples: (i) strain patterns show irregular variations in strain intensity with position around the fibers, and (ii) these variations and the overall strain intensity increase near the fiber tip in comparison with the fiber sides. In one case (at one end of Sample 4), past a certain strain level, strong deformation localization was noted near one of the two fiber ends. These data are shown in Fig. 7; at the higher stress level, strain is seen to concentrate within a band running roughly one fiber diameter away from the fiber tip, perpendicular to the fiber axis.

3.2.2. Stress. Photoelastic patterns collected from Sample 4 at the two stress levels of 7.8 and 9.5 $\mathrm{MPa}$ (corresponding to Fig. 7) are given in Fig. 8. Similar data from Sample 2, which contained no internal markers, are given in Fig. 9.

All specimens show large intensity differences across grain boundaries. While the overall trend is for each specimen to brighten as the load is increased, local intensity changes are not consistently monotonic with load. For example, two grains located to the right of the lower end of the fiber in Sample 4 are seen to darken (and exhibit new slip steps) as the load is increased from 7.8 to $9.5 \mathrm{MPa}$ (Fig. 8) (the darkening was ascertained not to be due to the specimen passing into the second fringe order [49]).

Slip steps at the surface are observable on all three specimens even in the low stress regions; these are remnants of deformation that occurred when cutting the tensile coupons from the diffusion bonded composite. During testing, slip bands of varying shades of grey developed within the individual grains; these are very clear in the large dark grain to the right of the upper end of the fiber in Sample 4 at $7.8 \mathrm{MPa}$, Fig. 8, or at the lower end of the fiber in Sample 2 (Fig. 9). These slip bands were particularly noticeable during the initial application of load, when they could be seen moving across the sample, and led to the additional slip steps that can be seen to appear in Sample 4 at a stress level of
9.5 MPa (Fig. 8), and in Sample 2 at stress levels of 7.4 and 9.0 MPa (Fig. 9).

\section{DISCUSSION}

\subsection{Theory}

To compare the data with predictions from isotropic continuum micromechanical analysis, two calculations were performed. The first is a finite element calculation, in which the elasto-plastic matrix is modeled as a von Mises isotropic solid. The second is the model based on Eshelby's equivalent inclusion calculation, which is essentially elastic in nature and is often used in micromechanical analysis of elasto-plastic composites.

4.1.1. Finite element calculations. All finite element modeling was performed using the software package ABAQUS (HKS Inc., Pawtucket, RI). A separate model was constructed for each experiment, using a large-strain quasistatic formulation.

Specimens were modeled as axisymmetric bodies whose diameters are equal to the width of the specimen. Meshes were constructed using Mathematica. All meshes were designed to have small elements in the vicinity of the fiber tip, where large strain gradients are expected; an example of a mesh is given in Fig. 10. Boundary conditions imposed that (i) symmetry lines along the left edge and bottom edge of the mesh as depicted in Fig. 10 remain stationary in the (horizontal) $r$ - and (vertical) $z$-directions respectively, (ii) the grip edge along the top be constrained to move a fixed distance in the $z$-direction, while remaining free to contract in the $r$-direction. The right-hand edge was stress-free and unconstrained. One model was run using four times as many elements as shown in the example mesh; the resultant stress and displacement fields did not differ significantly from the coarser mesh. It was therefore concluded that the mesh shown was sufficiently refined.

The models assumed that the titanium fiber was linearly elastic, with a modulus of $120 \mathrm{GPa}$ and a Poisson's ratio of 0.361 [54]. Based on tensile test data (Fig. 5) the silver chloride matrix was assumed to exhibit linear hardening after yield with a hardening coefficient of $42 \mathrm{MPa}$ and a yield stress of 7.4 $\mathrm{MPa}$. In the elastic regime, the modulus was taken as $29.5 \mathrm{GPa}$ and the Poisson's ratio as 0.33 [44].

The input parameter for the finite element calculations was applied displacement at the grip; how- 
ever, experimental measurements of applied load were far more accurate than measurements of applied displacement. In order to determine the appropriate input values for the applied displacement in the finite element calculation, the model was run twice. In the first run, the far-field values of the stress at the grip for small increments of the applied displacement were recorded. These data were used to construct a calculated load-displacement curve for the composite being modeled, which was then used to determine the set of applied displacements that correspond to the loads at which photographs had been taken during the experiment. Once the appropriate set of input displacements had been determined, the model was run again, recording displacement, strain, and stress for the entire specimen at the global strain levels corresponding to stress levels for which photographs had been taken during the experiment.

All the calculations assume that the matrix material is initially in a fully annealed, stress-free state. This assumption is supported by the observation, through examination of photoelastic patterns, that annealing removes residual stresses after diffusion bonding (see Section 2.1). Finite element predictions of strain contours are given next to experimental data in Figs 6 and 7.

Theoretical photoelastic images were computed from the FEM predictions for each strain increment. Formulae for the stress state as a function of thickness were obtained by third-order polynomial interpolation of the stress at each node. The photoelastic signal at each point was obtained by numerical integration of the following equation:

$$
\begin{aligned}
& \frac{\partial S}{\partial z}= \\
& \left(\begin{array}{cccc}
0 & 0 & 0 & 0 \\
0 & 0 & 0 & -C_{\sigma} \Delta \sigma \cos (2 \beta) \\
0 & 0 & 0 & C_{\sigma} \Delta \sigma \cos (2 \beta) \\
0 & C_{\sigma} \Delta \sigma \cos (2 \beta) & C_{\sigma} \Delta \sigma \cos (2 \beta) & 0
\end{array}\right) S
\end{aligned}
$$

where $S$ is the light beam vector:

$$
S=\left(s_{0}, s_{1}, s_{2}, s_{3}\right)^{\mathrm{T}}
$$

where $s_{0}$ represents the intensity of the light beam, and $s_{1}, s_{2}$, and $s_{3}$ represent the direction of polarization, with $s_{0}=\left(s_{1}^{2}+s_{2}^{2}+s_{3}^{2}\right)^{1 / 2}$. $\beta$ represents the orientation of the principal stresses resolved on the plane perpendicular to the light path, $\Delta \sigma$ is the difference between the principal stresses resolved on the plane perpendicular to the light path, $z$ is distance along the light path through the sample, and $C_{\sigma}$ is a material constant dependent on the wavelength of the light [49, 55].

Equation (2) was integrated across a "thick slice" cut through the center of the axisymmetric model, whose thickness corresponded to the thickness of the relevant specimen. The photoelastic constant used for the silver chloride was $3.4 \times 10^{-11} \mathrm{~m}^{2} \mathrm{~N}^{-1}$, as measured by Lum on identically processed specimens [56], a value within the range of values which have been reported by other researchers [44]. The Mathematica code used to produce the simulated photoelastic images is listed in Ref. [49]. Both circular and plane polarization micrographs under bright-field or dark-field conditions could be simulated from the same set of differential equations by varying the boundary conditions. Measured and calculated photoelastic stress patterns shown here are for circularly polarized light.

4.1.2. Eshelby equivalent inclusion calculation. Analytical models of the experimental specimens were also constructed using the Eshelby equivalent inclusion method, for comparison with experiment and FEM. Following the method described in Ref. [57] the cylindrical fiber was modeled as a prolate spheroid with its major axis equal to the fiber length, and its minor axis equal to the fiber radius. Plasticity is accounted for in the Eshelby equivalent inclusion models by assuming that the plastic strain is uniformly distributed in the matrix, so that it can be assimilated to an additional eigenstrain on the inclusion [3].

A Mathematica 2.2.2 package (incorporating both Mathematica and C code) was written (using Wolfram Research's MathLink 2.2.2) to calculate the Eshelby D-tensor for a prolate spheroid at points located in the matrix; the code is listed in Ref. [49]. This package uses the formulae for the Dtensor given in Ref. [1]. The eigenstrain was calculated by solving

$$
\begin{aligned}
& C_{i j k l}^{I}\left(\varepsilon_{k l}^{\mathrm{A}}+S_{k l m n}\left(\varepsilon_{m n}^{\mathrm{T}}-\varepsilon_{m n}^{\mathrm{p}}\right)-\varepsilon_{k l}^{\mathrm{p}}\right) \\
& \quad=C_{i j k l}^{\mathrm{M}}\left(\varepsilon_{k l}^{\mathrm{A}}+S_{k l m n}\left(\varepsilon_{m n}^{\mathrm{T}}-\varepsilon_{m n}^{\mathrm{p}}\right)-\varepsilon_{k l}^{\mathrm{T}}+\varepsilon_{k l}^{\mathrm{p}}\right.
\end{aligned}
$$

for the eigenstrain $\varepsilon^{\mathrm{T}}$, where $C^{\mathrm{I}}$ and $C^{\mathrm{M}}$ represent the stiffness tensors of the inclusion and the matrix, respectively, $\varepsilon^{\mathrm{A}}$ is the applied elastic strain, $\varepsilon^{\mathrm{p}}$ is the applied plastic strain, and $S$ is the Eshelby $S$ tensor of the inclusion, components of which are given in Ref. [3].

Since the applied displacements were not well known for the experimental specimens, the $\varepsilon^{\mathrm{p}}$ values used in the calculations were taken to be equal to the far-field strains of the corresponding finite element calculations minus the elastic strains, so that the boundary conditions of the two models are comparable. The plastic strain used in the Eshelby model was thus somewhat dependent on the predictions of the FEM calculations; however, as will be seen below, the samples examined were sufficiently long compared with the fibers for the stress and strain state along their ends to be, 


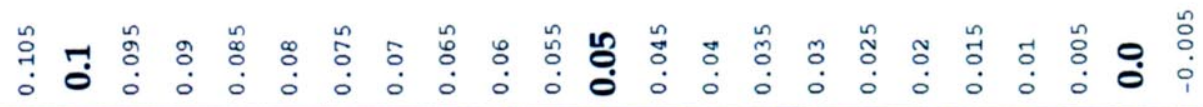
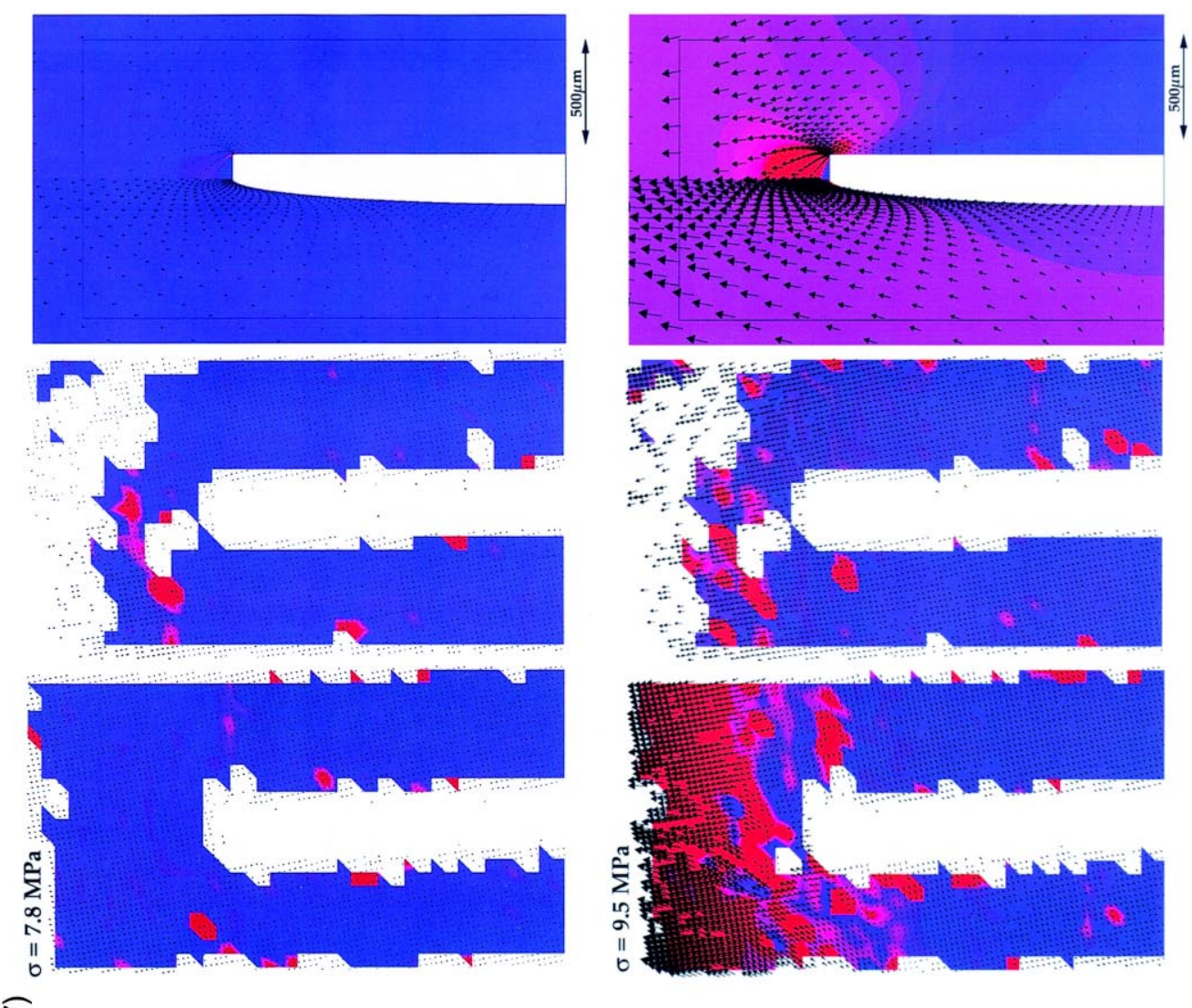

E
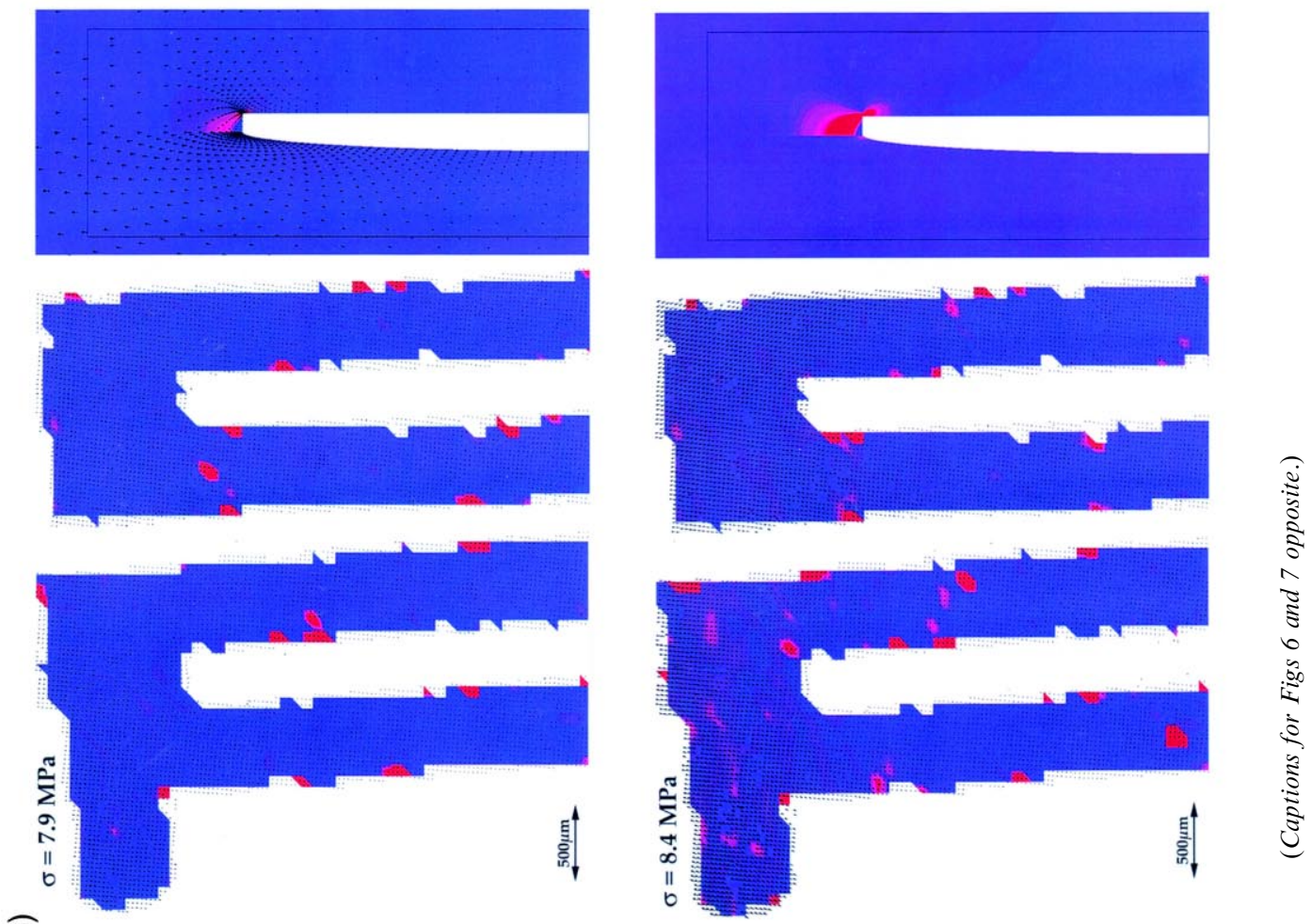

(6)

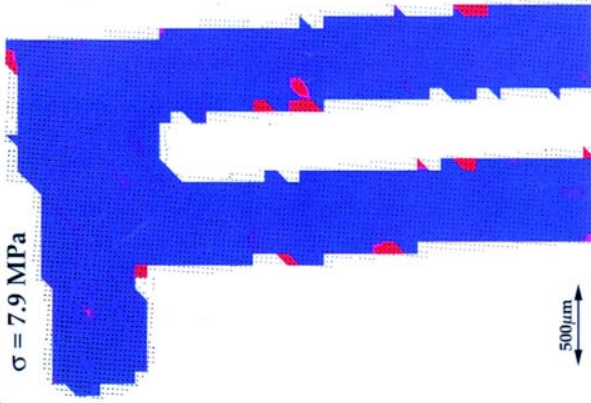


according to both models and in accordance with experiment, relatively uniform. Hence, far from the fibers near the sample ends, the local plastic strain is necessarily close in value to the plastic strain at that stress in the unreinforced matrix, with the implication that this procedure introduces no error in the estimation of $\varepsilon^{\mathrm{p}}$ used in the Eshelby model calculations.

The same code was used to calculate the simulated photoelastic photographs as was used for the FEM. The strain in the matrix was determined for a series of points situated on a quadrilateral mesh surrounding the ellipsoidal inclusion. A third order polynomial interpolation was used to calculate the value of the strain between the mesh points. The stress was calculated from the elastic strain in the Eshelby equivalent inclusion calculation according to:

$$
\sigma_{i j}=C_{i j k l}^{\mathrm{M}}\left(D_{k l m n} \varepsilon_{m n}^{\mathrm{T}}+\varepsilon_{k l}^{\mathrm{A}}\right)
$$

where $D$ is the (position dependent) Eshelby tensor in the matrix. Assuming axial symmetry, the photoelastic signal was then numerically integrated through the "thick slice" of the infinite Eshelby model which corresponded to the real specimen dimensions, as for the finite element calculations.

\subsection{Comparison of experiment with analysis}

4.2.1. Strain. Consider first the strain data for Samples 1 and 4, presented in Figs 6 and 7. We see that the experimental far-field strains, measured in the unreinforced portion of the matrix far from the fiber tip, are in fairly good agreement with both models. This indicates that the constitutive data and input displacements used in the calculations were reasonably accurate. Looking at the matrix region near the central portion of the fiber length (at the other end of the strain maps), we see that the finite element model satisfactorily predicts the strain in the matrix. The degree of overall reinforcement of the matrix provided by the fibers within the sample is therefore fairly well predicted by FEM. Comparatively, the Eshelby equivalent inclusion calculations show a much larger strain at the center of the fiber length. This is due to the fact that in this model, the plastic strain is assumed to be uniform over the whole matrix, rather than concentrated at the fiber ends as predicted by the finite element model and as observed experimentally.

At the fiber tip, neither model seems to really capture the essence of the experimental strain data. The finite element model shows a pronounced strain concentration about one fiber radius away from the fiber tip, with a small cap of very low strain material closer to the fiber end. The Eshelby calculation shows little strain concentration at the fiber end. The experimental data show a large and relatively diffuse region of slightly elevated strain for 
Sample 1 (Fig. 6); the same was found with Sample 3. Sample 4 (Fig. 7), shows a region of strain concentration similar to that of the FEM prediction at the upper end (middle pattern in the figure), and a large region of intense strain concentration somewhat beyond the fiber tip at the lower end (lefthand pattern). A comparison of strain data with the grain structure of the sample, which is made evident by the photoelastic patterns (see Fig. 8), shows that the region of intense strain concentration noted at the lower fiber end in Sample 4 corresponds overall with that delineated by two grains in that region.

An interesting characteristic of the data is the difference in the signal at the two ends of the fiber, a difference which is particularly striking in Sample 4 at higher applied loads. This is not duplicated in the models, since these assume from the outset that deformation is symmetric about the fiber half-length mid-plane. Clearly the initiation of increased deformation at one fiber end in comparison with the other end is linked with the difference in local orientation of surrounding crystals; the end more

$$
\sigma=7.8 \mathrm{MPa}
$$

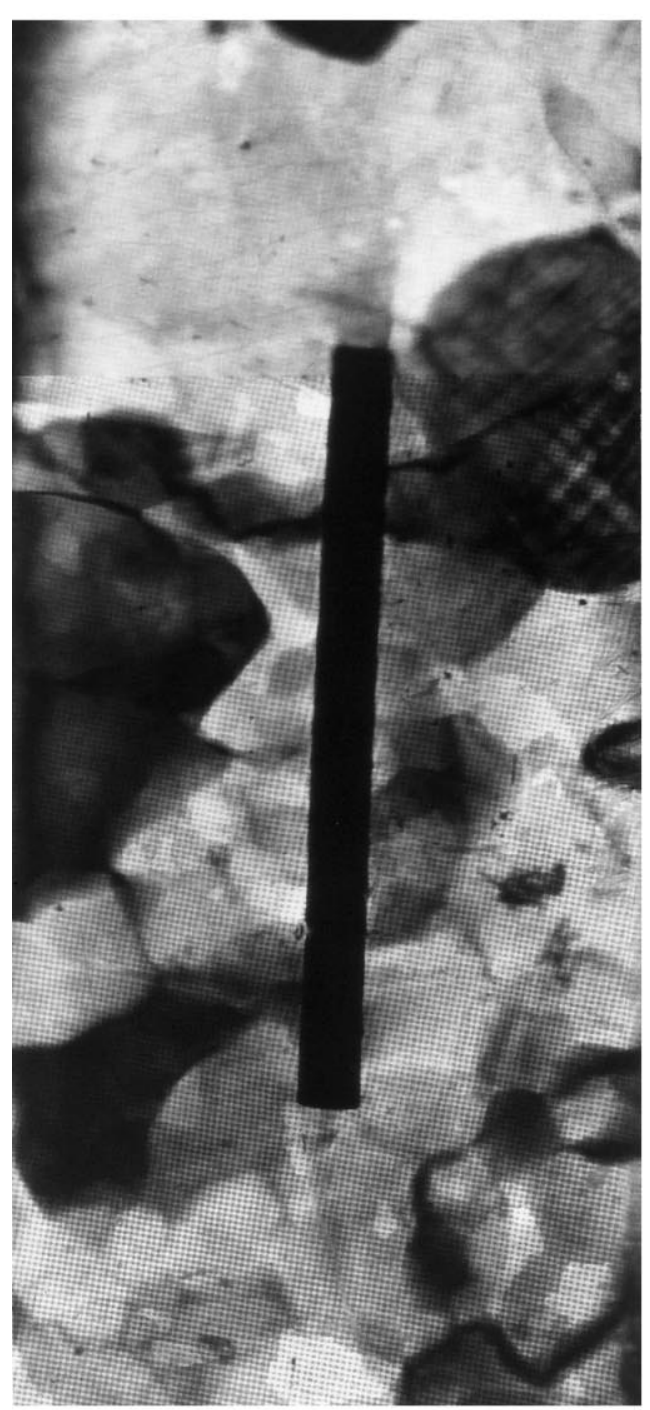

$$
\sigma=9.5 \mathrm{MPa}
$$

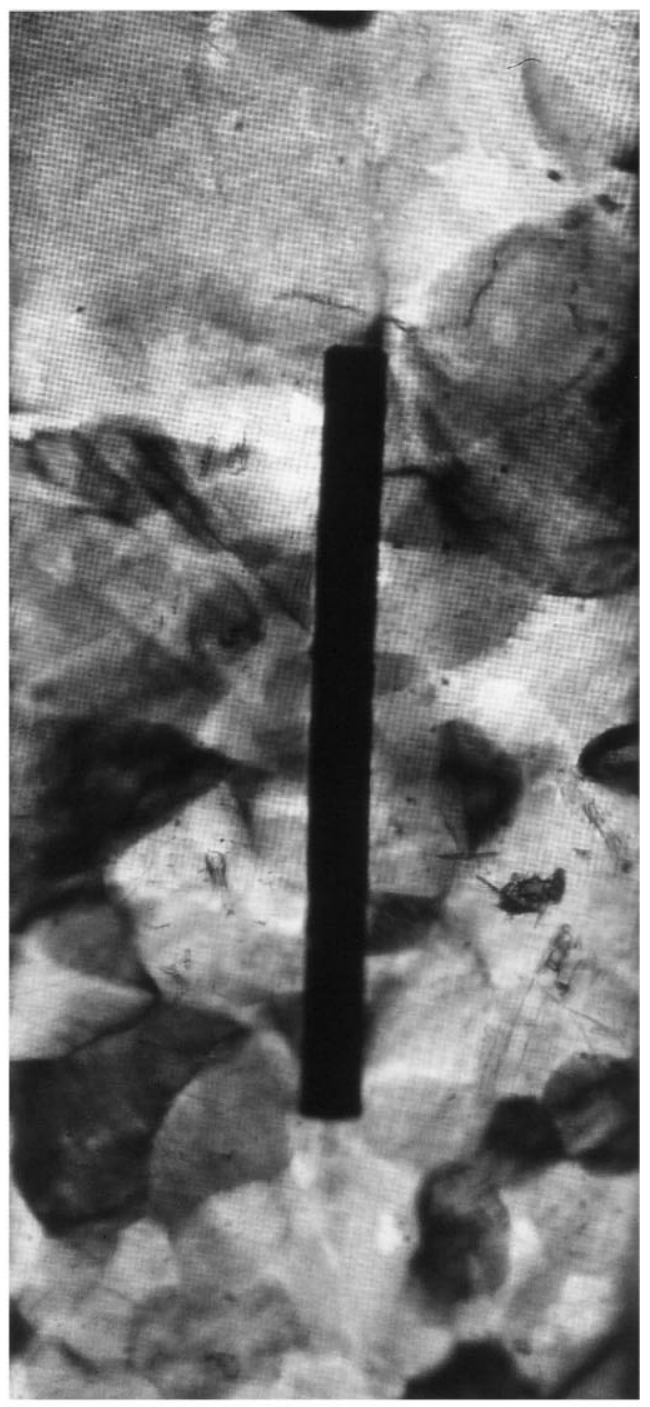

\section{$500 \mu \mathrm{m}$}

Fig. 8. Photoelastic stress patterns from Sample 4; at applied stresses of (left) 7.8, and (right) $9.5 \mathrm{MPa}$. Fiber diameter is $250 \mu \mathrm{m}$. 
prone to slip under the overall applied stress field yields first. Thereafter, it seems from the data that upon further straining, the difference between fiber ends persists, and can even be amplified (as seen on Sample 4, Fig. 7).

Overall, thus, the influence of crystallographic features of matrix plastic deformation on the deformation path around fibers is significant in the present samples, which feature grains of dimensions roughly commensurate with those of the fiber. This influence is sufficient to cause local strain distributions to deviate significantly from predictions of models based on isotropic continuum plasticity.
Comparatively, though, the finite element calculation captures far better the general features of matrix deformation around the inclusions than does the model based on the Eshelby calculation.

4.2.2. Stress. Photoelastic data are presented for Samples 4 and 2 in Figs 8 and 9, respectively. These images reveal matrix grains with great clarity. This means that significant differences exist in stress state from grain to grain within the ductile matrix as the sample deforms. These differences are also found to persist even near the fibers.

A comparison of predicted photoelastic patterns $\sigma=3.2 \mathrm{MPa}$

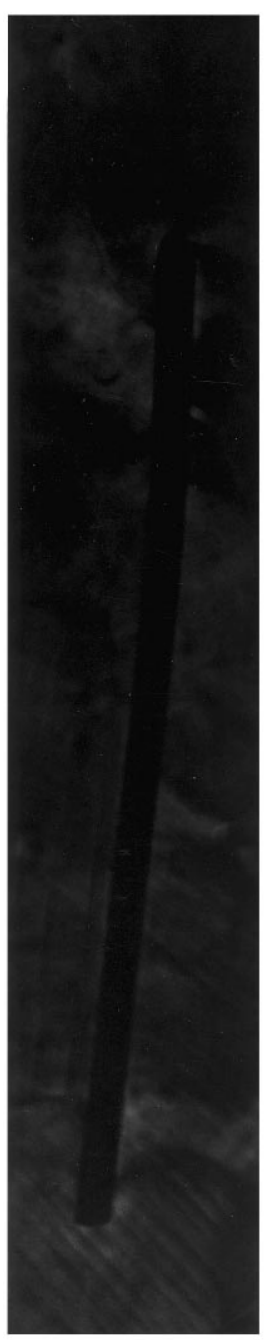

$$
\sigma=5.0 \mathrm{MPa}
$$

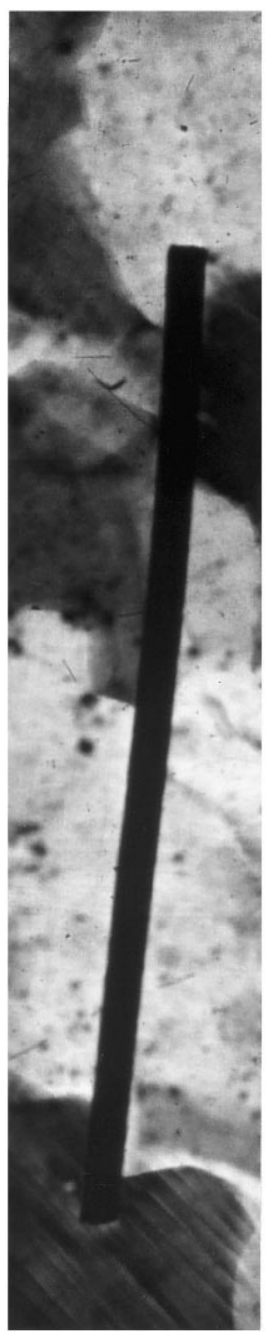

$$
\sigma=7.4 \mathrm{MPa}
$$

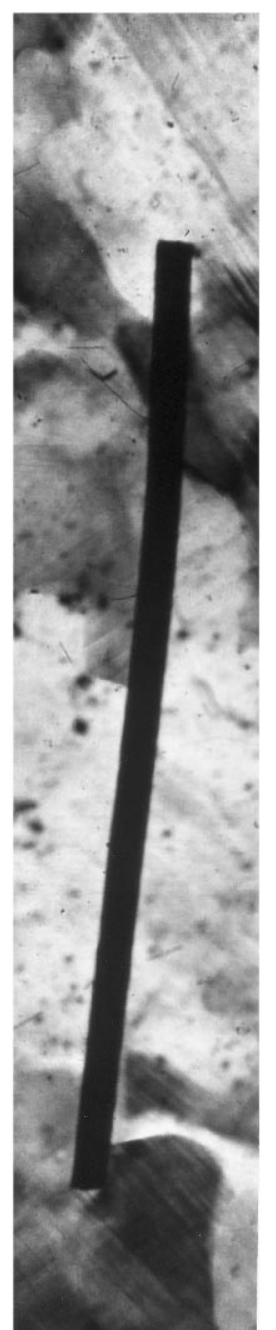

$\sigma=9.0 \mathrm{MPa}$

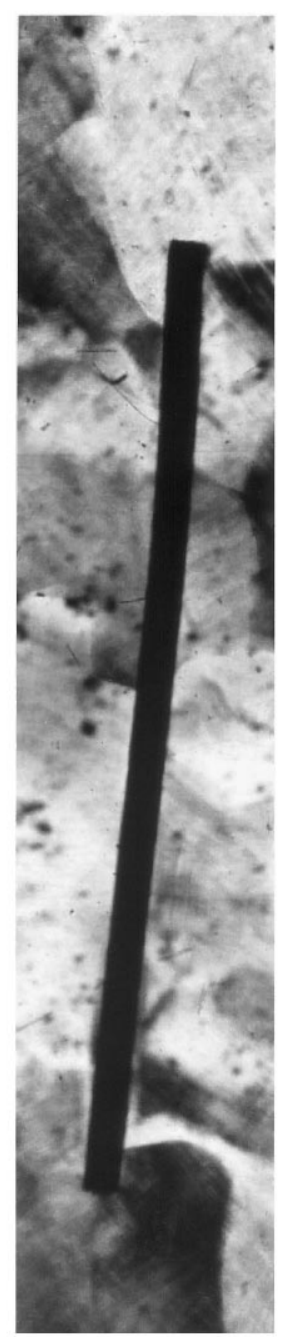

\section{$500 \mu \mathrm{m}$}

Fig. 9. Photoelastic stress patterns from Sample 2; at applied stresses of (from left to right) 3.2, 5.0, 7.4 and 9.0 MPa. Fiber diameter is $250 \mu \mathrm{m}$. 
for the region surrounding a fiber tip with data from Sample 2 is given in Fig. 11. The Eshelby model (far left) produces predictions which are very much at variance with the experimental data. At low applied stress (below about $4 \mathrm{MPa}$ ), this model was found to predict very little photoelastic signal, while experimentally there is some stress signal in the vicinity of the fiber tip even at these low applied stresses (Fig. 9). This disparity is probably due to the stress concentrations at the fiber corners, which are not present in the ellipsoidal inclusion assumed by the Eshelby model. At high stresses, the Eshelby model strongly overpredicts the signal, showing several fringe orders near the fiber (Fig. 11). This is due to the fundamentally elastic nature of this model, which causes it to predict much higher stresses than the real (elasto-plastic) matrix material can actually support at an applied stress of $9.5 \mathrm{MPa}$. The model predicts Von Mises stresses higher than $500 \mathrm{MPa}$, i.e. 70 times the yield stress and 50 times the applied stress, just ahead of the fiber tip for Sample 4. By contrast, the highest Von Mises stresses predicted by the finite element model are about $17 \mathrm{MPa}$, corresponding to 2.3 times the yield stress and less than twice the applied stress.

The isotropic von Mises finite element model is in

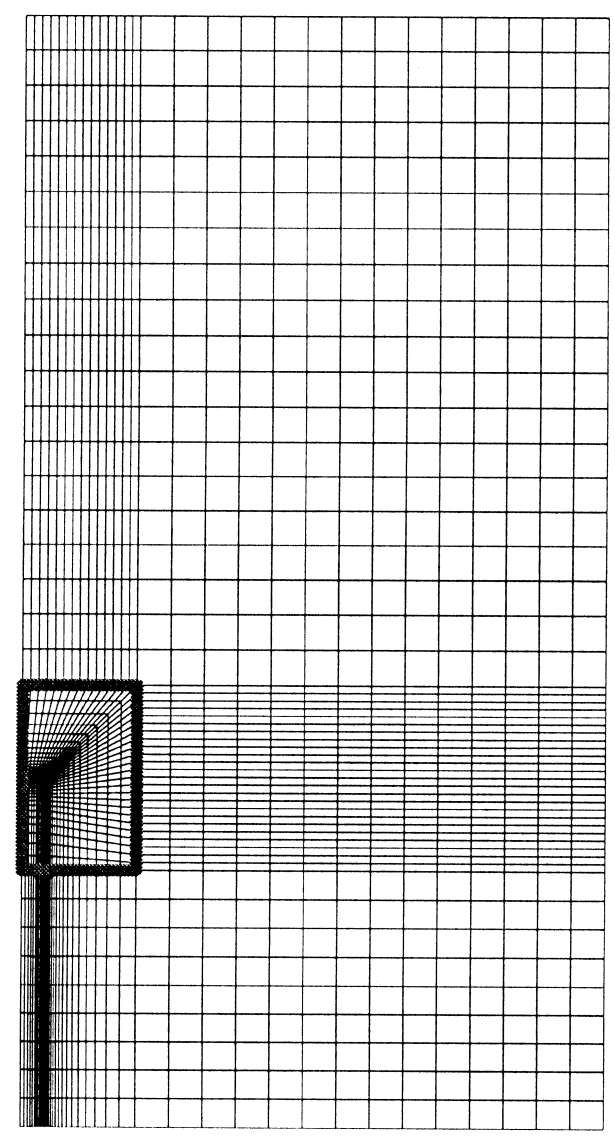

much closer agreement with experiment. Discrepancies arise in terms of the signal intensity in the photoelastic patterns, and also in terms of the location of regions of altered stress signal. For example, compare the calculated and observed photoelastic images for Sample 2 shown in Fig. 11: at one end of the fiber, one sees a very satisfying correspondence in the features of the FEM simulated image and the experimental data (taking the form of the two "mouse" ears at fiber end corners). At the other end the fiber appears to have far less of an effect on the photoelastic signal, which seems mostly dictated by the local distribution of grains and grain boundaries.

The most striking features of the experimental photoelastic images are in fact not the variations in photoelastic signal due to the fiber, but rather those due to microstructural features such as grain boundaries and slip bands. This effect is, for example, clearly shown by the build-up of stress at the grain boundary in Sample 2 (Fig. 9) at the lower end and to the right of the fiber. Some of this difference in signal strength is a consequence of the integrated nature of the photoelastic response; highly stressed regions which are spatially small can be masked by the grain response, which is present

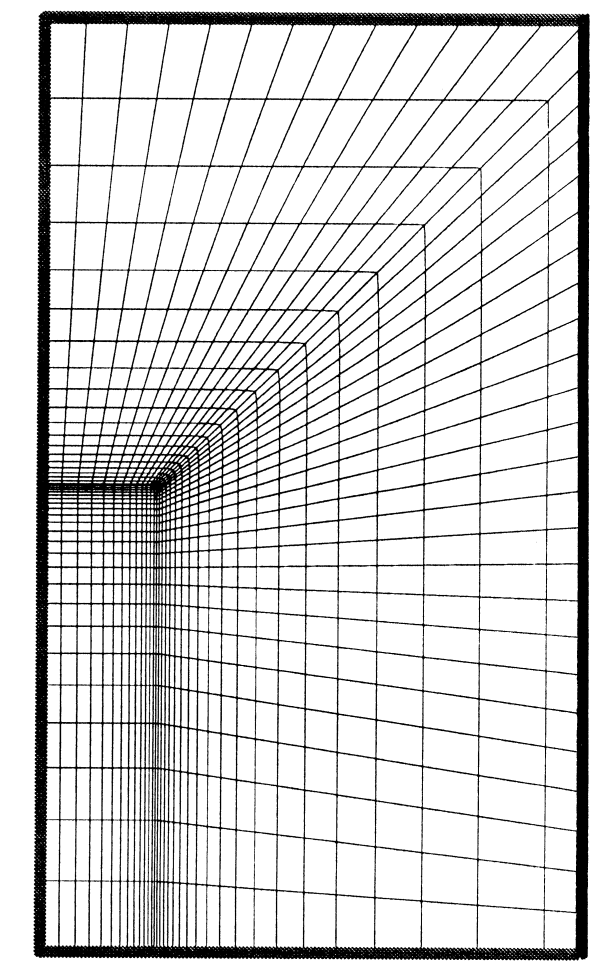

Fig. 10. Typical mesh used for finite element modeling. 
(a)
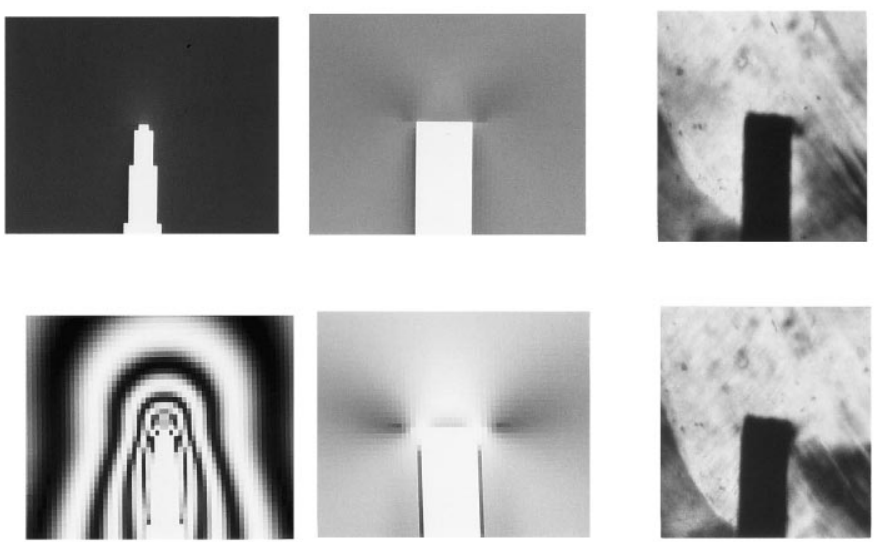

(c)

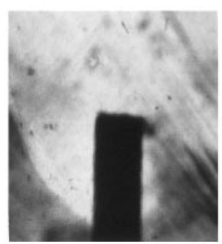

Fig. 11. Comparison of predicted and observed photoelastic patterns near the two tips of the fiber in Sample 2, at loads of 7.4 (top row) and 9.0 $\mathrm{MPa}$ (bottom row). (a) Eshelby equivalent inclusion calculation, (b) finite element analysis, (c) and (d) experimental (these last two images represent details of the two right-hand micrographs in Fig. 8). through the entire sample thickness. The samples are, however, about four fiber diameters in thickness; hence, although some masking of the signal arising from the fibers is to be expected, stress concentrations due to the fiber should nonetheless be clearly visible in the integrated signal. That the most obvious photoelastic features in these single fiber composites are due to the matrix microstructure thus underscores the importance of crystal plasticity to overall composite deformation.

4.2.3. Implications. Discrepancies between the experimental data and predictions based on the Eshelby equivalent inclusion calculation are strong and in fact not surprising. This is first and foremost due to the assumption implicit in the Eshelby calculation that matrix plastic deformation can be assimilated to a uniform eigenstrain. Variations in local strain are hence only caused by variations in local elastic strain, and plastic flow does not relieve stress concentrations. The latter are therefore overestimated while strain variations are underestimated. In essence, the Eshelby calculation fails to predict the essential features of microplastic deformation around the fibrous inclusions because it implicitly assumes that the fibers cause stress, not strain, concentrations in the surrounding elastoplastic matrix - whereas with plastic deformation one of course observes strain, not stress, concentrations. A second source of discrepancy with the Eshelby equivalent inclusion calculation is in the shape of reinforcement assumed: ellipsoidal inclusions do not account for the influence of sharp corners present on typical composite reinforcements such as chopped fibers, whiskers, or angular particles.

The data show far better agreement with predic- tions of finite element calculations; overall ranges of variation of stress and strain are adequately predicted, both near and far from the fiber ends. While local features of deformation at fiber ends are not regularly reproduced, some features, such as the local stress concentrations near fiber end corners, show good agreement. The finite element approach is therefore a far better (if imperfect) tool than the Eshelby calculation for micromechanical analysis of metal matrix composites, particularly when microscopic stress and strain distributions are under discussion.

Neither of the two models explored here takes into account the influence of metallurgical features having the same size scale as the reinforcement, such as individual grains and slip bands. Grain to grain variations in local stress and strain are manifestations of anisotropy, present in silver chloride for both plastic and elastic deformation modes. Given that the level of anisotropy in $\mathrm{AgCl}$ is commensurate with that of metals in terms of intensity (it is relatively high for elastic deformation, and somewhat lower for plastic deformation given the presence of pencil glide), an equally strong influence of grain structure on plastic flow is to be expected in metal matrix composites which show a similar relation of reinforcement dimensions to matrix grain size. The influence of grain boundaries is perhaps maximized in the present composites, since (i) with grains far smaller or far bigger than the reinforcement, one expects that the matrix can be far better represented as a continuum elasto-plastic material than in the present samples, and (ii) in the present samples, grains often extend across the thickness, such that there is somewhat less general constraint exerted by its neighbors on deformation of each grain than would be the case if grains were sur- 
rounded by neighbors in all three directions. The present work does, however, provide clear justification for current efforts towards modeling microplasticity with account of the influence exerted on plastic deformation by crystal plasticity and grain boundaries (e.g. [9, 58-63]).

\section{CONCLUSION}

A method allowing the measurement of both stress and strain distributions within bulk ductile material deforming by dislocational slip is presented. This method exploits the unique combination of transparency, stress birefringence, and room-temperature ductility of silver chloride, and combines the techniques of photoelasticity and marker tracking to gain information on the stress and strain state of the material both at low and high strains. As implemented here the technique features a spatial resolution near $30 \mu \mathrm{m}$ in strain measurements; however, the technique is only limited by the resolution of optical microscopes and can therefore feature a spatial resolution nearly ten times greater.

This technique is applied to the problem of an elasto-plastic ductile matrix containing a stiff fiber subjected to uniaxial stress applied parallel to the fiber axis. The experimental data are compared to two of the most common types of models used in metal matrix composite micromechanics: an analytical model based on the Eshelby equivalent inclusion calculation and a finite element model assuming an isotropic von Mises elasto-plastic matrix.

It is found that grain structure exerts a strong influence on both stress and strain distributions in the matrix of the composite, which features an average grain diameter on a par with the fiber length and diameter. The effects of the reinforcing fiber are visible in some respects, but are often overshadowed by the influence of crystal anisotropy and grain boundaries in the matrix. Neither model fully predicts significant features of the experimental data; however, the data are overall far better modeled by the finite element calculation based on isotropic von Mises plasticity.

At a more detailed level of comparison, the elevated strain region of the matrix in the vicinity of the fiber tip is found to be more diffuse and irregular than is predicted by the isotropic von Mises finite element model, while average strains far from the fiber ends are predicted satisfactorily. As regards the stress distribution near the fiber, photoelastic patterns predicted by the present finite element calculations show generally good agreement with experiment.

Overall, the data suggest that prediction of detailed features of the deformation and of stress distributions near inclusions will often require that the influence of grain boundaries and crystal plasticity be taken into account in micromechanical analysis.
Acknowledgements - We gratefully acknowledge support of this work from the National Science Foundation through awards DMR-9002558 and DMR-952775 under the supervision of Bruce MacDonald. The authors also would like to express their gratitude to Marc Finot and Subra Suresh of MIT for their substantial help and guidance in the finite element modeling portion of the present research, and to Dr Bhaskar Majumdar of the Wright Patterson Air Force Base for very helpful discussions and suggestions in connection with this work.

\section{REFERENCES}

1. Mura, T., Micromechanics of Solids. Martinus Nijhoff, The Hague, 1982.

2. Taya, M. and Arsenault, R. J., Metal Matrix Composites: Thermomechanical Behavior. Pergamon Press, Oxford, 1989.

3. Clyne, T. W. and Withers, P. J., An Introduction to Metal Matrix Composites. Cambridge University Press, Cambridge, 1993.

4. Christman, T., Needleman, A., Nutt, S. and Suresh, S., Mater. Sci. Engng, 1989, A107, 49.

5. Christman, T., Needleman, A. and Suresh, S., Acta metall., 1989, 37, 3029.

6. Dvorak, G. J., in Metal Matrix Composites: Mechanisms and Properties, ed. R. K. Everett and R. J. Arsenault. Academic Press, Boston, 1991, pp. 1-78.

7. Tvergaard, V., in Proc. Conf. Metal Matrix Composites-Processing, Microstructure and Properties, 12th Riso Int. Symp. on Materials Science, Risø, Denmark, ed. N. Hansen, D. Juul-Jensen, T. Leffers, H. Lilholt, T. Lorentzen, A. S. Pedersen, O. B. Pedersen and B. Ralph. Risø National Laboratory, Roskilde, Denmark, 1991, pp. 173-188.

8. Suresh, S. and Brockenbrough, J., in Fundamentals of Metal Matrix Composites, ed. S. Suresh, A. Mortensen and A. Needleman. ButterworthHeinemann, Stoneham, MA, 1993, pp. 174-190.

9. McHugh, P. E., Asaro, R. J. and Shih, C. F., in Fundamentals of Metal Matrix Composites, ed. S. Suresh, A. Mortensen and A. Needleman. Butterworths, Boston, 1993, pp. 139-157.

10. Martin, J. W., in Micromechanisms in ParticleHardened Alloys. Cambridge University Press, Cambridge, UK, 1980, pp. 72-79.

11. Ochiai, S. (ed.), Mechanical Properties of Metallic Composites. Dekker, New York, 1994.

12. Wilkinson, A. J., Gonzalez, G. and Dingley, D. J., J. Microsc., 1993, 126, 255.

13. Isaacs, J. A. and Mortensen, A., Metall. Trans., 1992, 23A, 1207.

14. Kim, C. T., Lee, J. K. and Plichta, M. R., Metall. Trans., 1990, 21A, 673.

15. Barlow, C. Y. and Hansen, N., Acta metall. mater., 1995, 43, 3633.

16. Barlow, C. Y. and Liu, Y. L., Acta mater., 1998, 46, 5807.

17. Prangnell, P. B. and Stobbs, W. M., in Proc. Conf. 12th Risø Int. Symp. on Metallurgy and Materials Science: Metal Matrix Composites-Processing, Microstructure and Properties, Roskilde, Denmark, ed. N. Hansen, D. Juul-Jensen, T. Leffers, H. Lilholt, T. Lorentzen, A. S. Pedersen, O. B. Pedersen and B. Ralph. Risø National Laboratory, Roskilde, Denmark, 1991, pp. 603-610.

18. Prangnell, P. B., Downes, T., Stobbs, W. M. and Whithers, P. J., Acta metall. mater., 1994, 42, 3425.

19. Chawla, K. K. and Metzger, M., J. Mater. Sci., 1972, 7, 34.

20. Chawla, K. K. and Metzger, M., Metall. Trans., 1977, 8A, 1681 . 
21. Kuntz, T. D., Wadley, H. N. G. and Black, D. R., Metall. Trans., 1993, 24A, 1117.

22. Kendig, K. L., Soboyejo, W. O. and Miracle, D. B., Scripta metall. mater., 1995, 32, 669.

23. Cohen, J. B., Powder Diffraction, 1986, 1, 15

24. Allen, A. J., Bourke, M. A. M., Dawes, S. Hutchings, M. T. and Withers, P. J., Acta metall. mater., 1992, 40, 2361.

25. Bourke, M. A. M., Goldstone, J. A., Stout, M. G. and Needleman, A., in Fundamentals of Metal Matrix Composites, ed. S. Suresh, A. Mortensen and A. Needleman. Butterworth-Heinemann, Stoneham, MA, 1993, pp. 61-80.

26. Wilkinson, A. J., Mater. Sci. Engng, 1991, A135, 189.

27. Wilkinson, A. J. and Dingley, D. J., Acta metall mater., 1992, 40, 3357.

28. Davidson, D. L., Metall. Trans., 1987, 18A, 2115.

29. Davidson, D. L., Metall. Trans., 1991, 22A, 113.

30. Davidson, D. L., Metall. Mater. Trans., 1997, 28A, 1297.

31. Davidson, D. L. and McClung, R. C., Int. J. Fracture, 1997, 84, 81

32. Poole, W. J., Kocks, U. F., Bolmaro, R. E. and Embury, J. D., in Proc. Conf. Metal Matrix Composites-Processing, Microstructure and Properties, 12th Riso Int. Symp. on Materials Science, Roskilde, Denmark, ed. N. Hansen, D. Juul-Jensen, T. Leffers, H. Lilholt, T. Lorentzen, A. S. Pedersen, O B. Pedersen and B. Ralph. Risø National Laboratory, Roskilde, Denmark, 1991, pp. 587-593.

33. Özturk, T., Poole, W. J. and Embury, J. D., Mater Sci. Engng, 1991, A148, 175.

34. Allais, L., Bornert, M., Bretheau, T. and Caldemaison, D., Acta metall. mater., 1994, 42, 3865.

35. Maruyama, B., Shaw, L. L., Waterbury, M. C. and Miracle, D. B., in Proc. Tenth International Conference on Composite Materials, Whistler, British Columbia, Canada, ed. A. Poursartip and K. Street. Woodhead, Cambridge, 1995, pp. II-75-II-82.

36. Liu, Y. L. and Fischer, G., in Proc. Eleventh International Conference on Composite Materials, Gold Coast, Queensland, Australia, ed. M. L. Scott. Woodhead, Cambridge, 1997, pp. III-1-III-9.

37. Liu, Y. L. and Fischer, G., Scripta mater., 1997, 36 1187

38. Marschall, D. B., Morris, W. L., Cox, B. N., Graves, J., Porter, J. R., Kouris, D. and Everett, R. K., Acta metall. mater., 1994, 42, 2657.

39. Nye, J. F., Proc. R. Soc. Lond., 1949, A198, 190

40. Gmelin and Institut (ed.), Gmelins Handbuch der Anorganischen Chemie: Silber B2. Verlag Chemie, 1972 .

41. Gmelin and Institut (ed.), Gmelins Handbuch der Anorganischen Chemie: Silber B1. Verlag Chemie, 1971.

42. Frieser, H., Haase, G. and Klein, E. (ed.), Die Grundlagen der Protographischen Prozess mit Silberhalogeniden, vols 1-3. Akademische Verlagsgesellschaft, 1968.

43. Mees, E. K. and James, T. H., The Theory of the Photographic Process, 3rd edn. Macmillan, New York, 1966.
44. Javornicky, J., in Photoplasticity. Elsevier Scientific, Amsterdam, 1974, pp. 212-237.

45. Sprackling, M. T., The Plastic Deformation of Simple Ionic Crystals. Academic Press, New York, 1976.

46. Moser, F., Nail, N. R. and Urbach, F., J. Phys. Chem. Solids, 1959, 9, 217.

47. Moeller, R. D., Schonfeld, F. W., Tipton, C. R. and Waber, J. T., Trans. Am. Soc. Metals, 1951, 43, 39

48. Taylor, N., Dunand, D. C. and Mortensen, A., Acto metall. mater., 1993, 41, 955.

49. Earhart, E. J., Experimental investigation of stress and strain fields in a ductile matrix surrounding an elastic inclusion. Ph.D., Massachusetts Institute of Technology, 1997.

50. Sirkis, J. S. and Lim, T. J., Exp. Mech., 1991, 31, 382.

51. Haralick, R. M., Sternberg, S. R. and Zhuang, X., IEEE Trans. Pattern Anal. Mach. Intell., 1987, PAMI9, 532 .

52. Standard Test Methods for Tension Testing of Metallic Materials (Metric), Standard E 8M-95a. ASTM Annual Book of Standards 03.01. American Society for Testing of Materials, 1995, pp. 77-93.

53. Corvasce, F., Lipinski, P. and Berveiller, M., in Proc. Conf. IUTAM Symposium, Troy, $N Y$, ed. G. J. Dvorak. Springer, New York, 1990, pp. 389-408.

54. Boyer, H. E. and Gall, T. L. (ed.), Metals Handbook-Desktop Edition. ASM, Metals Park, OH, 1985 , pp. 2-16.

55. Theocaris, P. S. and Gdoutos, E. E., Matrix Theory of Photoelasticity. Springer, Berlin, 1979, pp. 35, 63, 133-142.

56. Lum, J. W., Prediction and analysis of matrix stresses near broken fibers in titanium fiber-reinforced silver chloride, B.Sc., Department of Materials Science and Engineering, Massachusetts Institute of Technology, 1994.

57. Withers, P. J., Smith, A. N., Clyne, T. W. and Stobbs, W. M., in Fundamental Relationships between Microstructure \& Mechanical Properties of MetalMatrix Composites, Proc. Conf. Fundamental Relationships Between Microstructure \& Mechanical Properties of Metal-Matrix Composites, ed. P. K. Liaw and M. N. Gungor. The Minerals, Metals \& Materials Society, 1990, pp. 225-240.

58. Yao, Z. and Wagoner, R. H., Acta metall. mater., 1993, 41, 451.

59. Tungatt, P. D. and Humphreys, F. J., in Proc. Conf. Deformation of Polycrystals: Mechanisms and Microstructures: 2nd Riso Symposium on Metallurgy and Materials Science, Risø National Laboratory, Denmark, 1981, pp. 393-398.

60. Bretheau, T., Mussot, P. and Rey, C., Trans. ASME J. Engng Mater. Technol., 1984, 106, 304.

61. Needleman, A. and Tvergård, V., Trans. ASME J. Appl. Mech., 1993, 60, 70.

62. McHugh, P. E., Asaro, R. J. and Shih, C. F., Acta metall. mater., 1993, 41, 1461.

63. Needleman, A., Suresh, S. and Tvergård, V., in Local Mechanics Concepts for Composite Material Systems, ed. J. N. Reddy and K. L. Reifsnider. Springer, Berlin, 1992, pp. 199-213. 